\title{
Iodine intake is positively associated with iodine knowledge in women of childbearing age
}

\author{
S.M. O’Kane, L.K. Pourshahidi, K.M. Farren, M.S. Mulhern, J.J. Strain and A.J. Yeates \\ Northern Ireland Centre for Food and Health (NICHE), University of Ulster, Cromore Road, Coleraine, Co. \\ Londonderry, BT52 1SA, UK
}

This abstract was awarded the student prize for best oral original communication.

Iodine is required for the production of thyroid hormones which are essential during pregnancy and infancy for brain and neurological development ${ }^{(1)}$. It is important that women of childbearing age have an adequate iodine intake to ensure sufficient iodine stores in the thyroid pre-conception ${ }^{(2)}$. Recent evidence has suggested that women of childbearing age in the UK are iodine deficient and concern has been expressed regarding current dietary iodine intake ${ }^{(3,4)}$. The most recent National Diet and Nutrition Survey (NDNS) reported that women aged 19-65 years have a median daily iodine intake of $126 \mu \mathrm{g}$ which is $10 \%$ below the reference nutrient intake (RNI) ${ }^{(5)}$. The aim of the present study was to assess the association between iodine intake and iodine knowledge among women of childbearing age.

Females (18-45 years) were asked to complete a 30-item questionnaire which was administered online using SurveyMonkey ${ }^{\circledR}$ between February and March 2015. The questionnaire collected demographic information and assessed knowledge of iodine. The final section of the questionnaire collected information on the consumption of iodine-rich foods and was adapted from the Glasgow Iodine Food Frequency Questionnaire ${ }^{(6)}$ to include eggs. Daily iodine intake was estimated by combining the number of servings with average portion size and iodine content per serving ${ }^{(7,8)}$.

To date, a total of 431 respondents have completed the questionnaire (52\% 18-25 years; $8 \%$ current smokers; $4 \%$ pregnant; $1 \%$ breastfeeding). The median $\left(25-75^{\text {th }}\right.$ percentile) daily iodine intake was $152 \mu \mathrm{g}(98-212 \mu \mathrm{g})$. Almost half of respondents $(44 \%)$ had intakes below the RNI of $140 \mu \mathrm{g} /$ day. A significantly greater proportion of younger respondents (18-30 years) had an iodine intake below the RNI than older respondents $(31-45$ years) (31\% vs. $14 \% ; P=0.034)$. Similar to the latest NDNS findings ${ }^{(5)}, 13 \%$ of respondents had a daily iodine intake below the lower reference nutrient intake. Milk provided the largest contribution to daily iodine intake (45\%). Other dairy products, fish and eggs made smaller contributions to daily iodine intake; $23 \%, 18 \%$ and $13 \%$ respectively. Those with a low daily milk intake $(<200 \mathrm{mls})$ had significantly lower daily iodine intake than those with a higher milk intake $(142 \mu \mathrm{g} / \mathrm{d}$ vs. $167 \mu \mathrm{g} / \mathrm{d} ; P=0.038)$. Iodine containing supplements were only consumed by $3 \%$ of respondents and iodised salt was purchased by only $7 \%$ of respondents. Iodine intake was positively correlated with iodine knowledge $(P=0 \cdot 014)$. Those with a low level of iodine knowledge had a significantly lower daily iodine intake than those with a moderate to high level of iodine knowledge $(152 \mu \mathrm{g} / \mathrm{d}$ vs. $166 \mu \mathrm{g} / \mathrm{d} ; P=0 \cdot 046)$. Iodine intake was not significantly related to respondent's level of education, their awareness of the current public health concern about iodine deficiency, or whether or not they had previously received information on iodine nutrition.

These findings demonstrate a clear association between iodine intake and level of iodine knowledge, albeit estimated from selfreported data. The implementation of effective education programmes should be considered to increase dietary iodine intakes among a group vulnerable to the effects of iodine deficiency.

Ethical approval was granted from the Ulster University, School of Biomedical Sciences Ethics Filter Committee and the study was conducted according to the guidelines laid down in the Declaration of Helsinki.

1. Zimmermann (2009) Endocr Rev. 30 (4): 376-408.

2. Zimmermann (2009) Am J Clin Nutr. 89 (2): 668-672.

3. Lampropoulou et al. (2012) Proc Nutr Soc. 71 (OCE2) E143.

4. Rayman et al. (2008) Proc Nutr Soc. 67 (OCE8) E399.

5. Bates et al. (2014) National Diet and Nutrition Survey.

6. Combet \& Lean (2014) J Hum Nutr Diet. 27 (6): 599-605.

7. Food Standards Agency (2002) McCance and Widdowson's The Composition of Foods.

8. Food Standards Agency (2002) Food Portion Sizes. 